Pacific Journal of Mathematic 


\title{
ON THE FIRST AND THE SECOND CONJUGATE POINTS
}

\author{
W. J. KIM
}

\begin{abstract}
Three properties of conjugate points and extremal solutions of an $n$ th-order linear ordinary differential equation are discussed. Also, a connection between the zero distribution and the factorization of an $n$ th-order differential operator in the interval $\left(a, \eta_{2}(a)\right)$ is established.
\end{abstract}

1. Introduction. We shall be concerned with the $n$ th-order differential equation

$$
L y=\sum_{k=0}^{n} p_{k}(x) y^{(k)}=0
$$

where the coefficients are real-valued functions which are continuous on an interval $I$ and $p_{n}(x) \neq 0, x \in I$. A differential equation of the form (1.1) is called nonsingular on $I$. A solution $y$ of (1.1) is said to have a zero of order $k$ at $c \in I$ if $y(c)=y^{\prime}(c)=\cdots=y^{(k-1)}(c)=0$; if in addition $y^{(k)}(c) \neq 0$, we say that $y$ has a zero of order exactly $k$ at $c$. A zero of order exactly one is called simple. The $m$ th conjugate point $\eta_{m}(a)$ of a point $a \in I$ is the smallest number $b>a, b \in I$, such that there exists a nontrivial solution of (1.1) which vanishes at $a$ and has $n+m-1$ zeros (counting multiplicities) on $[a, b][6]$. Obviously, we have the relation $\eta_{1}(a) \leqq \eta_{2}(a) \leqq \cdots$. A nontrivial solution of (1.1) which has $n$ zeros on $\left[a, \eta_{1}(a)\right]$ is called an extremal solution for the interval $\left[a, \eta_{1}(a)\right]$. A nontrivial solution of (1.1) is said to have an $i_{1}-i_{2}-\cdots-i_{j}$ distribution of zeros on $I$ if it has a zero of order $i_{k}$ at $x_{k} \in I, x_{1}<x_{2}<\cdots<x_{j}, k=1,2, \cdots, j$.

So far as the study of zero distribution of solutions $[1-5,8-11,14]$ is concerned, it is convenient to divide the problem into two cases: $\eta_{1}(a)=\eta_{2}(a)$ and $\eta_{1}(a)<\eta_{2}(a)$. In a recent paper, Gustafson [2] obtained an interesting result for the case $\eta_{1}(a)=\eta_{2}(a)$. Evidently, $\eta_{1}(a)<\eta_{2}(a)$ for any second-order differential equation of the form (1.1). However, for higher-order equations both cases $\eta_{1}(a)=\eta_{2}(a)$ and $\eta_{1}(a)<\eta_{2}(a)$ occur. For example, $\eta_{1}(a)=\eta_{2}(a)=\eta_{3}(a)$ for the equation $y^{(v)}+10 y^{\prime \prime}+9 y=0[1]$, while $\eta_{1}(a)<\eta_{2}(a)$ for

$$
\left(r y^{\prime \prime}\right)^{\prime \prime}-p y=0, \quad r>0, \quad p>0, \quad r \in C^{\prime \prime}, \quad p \in C,
$$


according to a result of Leighton and Nehari [6]. Other equations with the property

$$
\eta_{1}(a)<\eta_{2}(a)
$$

have been observed by Peterson [10].

Suppose Eq. (1.1) has an extremal solution for $\left[a, \eta_{1}(a)\right]$. Then it is well-known that (1.1) has an extremal solution for $\left[a, \eta_{1}(a)\right]$ which does not vanish on $\left(a, \eta_{1}(a)\right)$ [12]. Of particular interest is the equation which has the property

$\left(\mathrm{P}_{2}\right) \quad$ No extremal solution for $\left[a, \eta_{1}(a)\right]$ vanishes on $\left(a, \eta_{1}(a)\right)$.

For example, it can be easily shown that $y^{\prime \prime \prime}+y=0$ and $y^{\prime \prime \prime}-y=0$ have the property $\left(\mathrm{P}_{2}\right)$. In fact, every extremal solution of $y^{\prime \prime \prime}+y=0$ has a 2-1 distribution of zeros. On the other hand, every extremal solution of $y^{\prime \prime \prime}-y=0$ has a 1-2 distribution of zeros. These two equations also have the property $\left(\mathrm{P}_{1}\right)$.

As it turns out, closely connected with $\left(P_{1}\right)$ and $\left(P_{2}\right)$ is the property

$\left(\mathrm{P}_{3}\right)$ There do not exist two (not necessarily distinct) extremal solutions for $\left[a, \eta_{1}(a)\right]$ with zero distributions $(n-k)-k$ and $(n-k-1)-(k+1)$, respectively, where $k$ is a fixed number, $1 \leqq k \leqq n-2$.

In $\$ 2$ we prove that $\left(P_{3}\right)$ implies $\left(P_{1}\right)$ and $\left(P_{2}\right)$. Conversely, $\left(P_{1}\right)$ and $\left(\mathrm{P}_{2}\right)$ taken together imply $\left(\mathrm{P}_{3}\right)$. Moreover, we shall show that in general $\left(\mathrm{P}_{1}\right)$ neither implies nor is implied by $\left(\mathrm{P}_{2}\right)$. As the last result of this section we shall exhibit a class of differential equations which has the properties $\left(\mathrm{P}_{1}\right),\left(\mathrm{P}_{2}\right)$ and $\left(\mathrm{P}_{3}\right)$.

In $\$ 3$ we assume $\left(P_{1}\right)$ and investigate the zero distribution of solutions on the intervals $\left[a, \eta_{1}(a)\right]$ and $\left(a, \eta_{2}(a)\right)$, and their consequences. In particular, we discuss a connection between the zero distribution and the factorization of (1.1) on the interval $\left(a, \eta_{2}(a)\right)$.

In the sequel we shall have an occasion to use the function $w\left(x ; x_{1}^{\left[k_{1}\right]}, x_{2}^{\left[k_{2}\right]}, \cdots, x_{p}^{\left[k_{p}\right]}\right)$ defined and used in [5]. Let $y_{1}, y_{2}, \cdots, y_{n}$ be $n$ linearly independent solutions of (1.1). Then the function $w$ is defined by 
$w\left(x ; x_{1}^{\left[k_{1}\right]}, x_{2}^{\left[k_{2}\right]}, \cdots, x_{p}^{\left[k_{p}\right]}\right)$

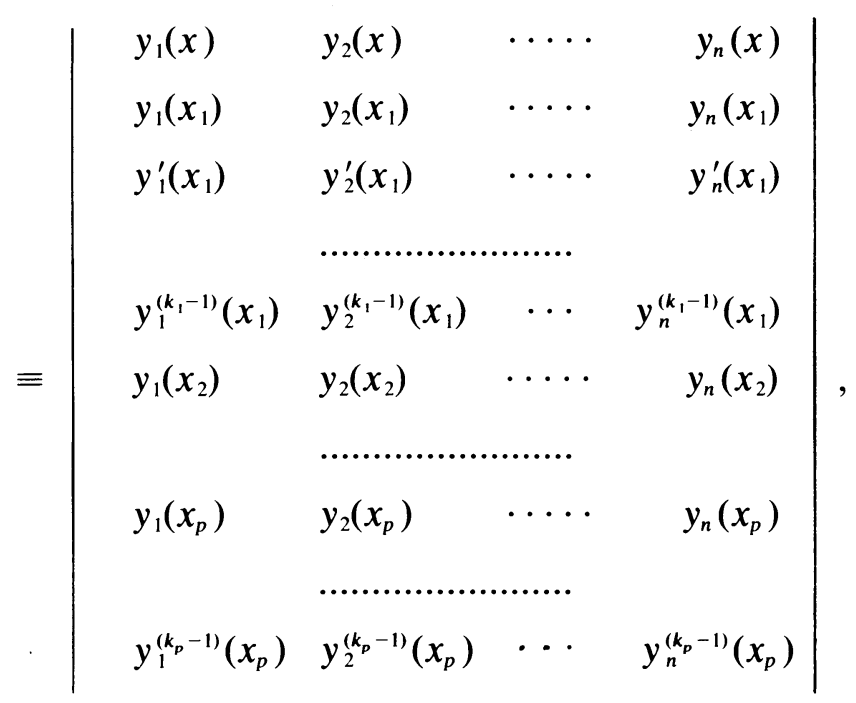

$1 \leqq p \leqq n-1, k_{1}+k_{2}+\cdots+k_{p}=n-1$. Obviously, this function $w$ is a solution of (1.1) with a zero of order $k_{i}$ at $x_{i}, i=1,2, \cdots, p$. Moreover, it is continuous function of $x_{1}, x_{2}, \cdots, x_{p}$.

2. Properties $\left(P_{1}\right),\left(P_{2}\right)$ and $\left(P_{3}\right)$. Suppose $(1.1)$ has an extremal solution $Y$ for $\left[a, \eta_{1}(a)\right]$ with an $i_{1}-i_{2}-\cdots-i_{j}$ distribution of zeros, i.e., $Y$ has a zero of order $i_{k}$ at $x_{k}, k=1,2, \cdots, j, i_{1}+i_{2}+\cdots+i_{j}=$ $n, a=x_{1}<x_{2}<\cdots<x_{j}=\eta_{1}(a)$. Numerous results have been obtained for the zero distribution of $Y[2,5,9,10,12]$. Of particular importance in this section is the following result which will be frequently referred to in the proofs.

THEOREM 2.1 [5]. If Y has a zero of order exactly $i_{m}$ at $x_{m}, 2 \leqq m \leqq$ $j-1$, then (1.1) has an extremal solution for $\left[a, \eta_{1}(a)\right]$ with an $i_{1}-\cdots-$ $i_{m-1}-\left(i_{m}-1\right)-i_{m+1}-\cdots-i_{j}$ distribution of zeros and an additional zero at an arbitrary point $\xi \in\left[a, \eta_{1}(a)\right]$.

A simple application of this theorem shows that $\left(\mathrm{P}_{3}\right)$ implies $\left(P_{2}\right)$. This result can then be used to prove that $\left(P_{3}\right)$ also implies $\left(P_{1}\right)$. On the other hand, if $(1.1)$ does not satisfy $\left(P_{3}\right)$, it is easily confirmed that $(1.1)$ must violate either $\left(P_{1}\right)$ or $\left(P_{2}\right)$.

THEOREM 2.2. Eq. (1.1) has the property $\left(\mathrm{P}_{3}\right)$ if and only if it satisfies $\left(\mathrm{P}_{1}\right)$ and $\left(\mathrm{P}_{2}\right)$.

We shall illustrate by means of examples that in general $\left(P_{1}\right)$ neither implies nor is implied by $\left(P_{2}\right)$. The nonsingular equation 


$$
y^{\prime \prime \prime}-\frac{6 \sin x \cos x\left(\cos ^{2} x-\sin ^{2} x\right)}{3 \sin ^{2} x \cos ^{2} x-2} y^{\prime \prime}
$$

$$
-\frac{9 \sin ^{2} x \cos ^{2} x+14}{3 \sin ^{2} x \cos ^{2} x-2} y^{\prime}=0
$$

has as a fundamental set of solutions $\sin ^{2} x \cos x, \cos ^{2} x \sin x$, and 1 $[1,13]$. The Wronskian $W$ of these three solutions is given by $W=$ $3 \sin ^{2} x \cos ^{2} x-2<0$, and the corresponding adjoint equation

$(2.1)^{*}$

$$
v^{\prime \prime \prime}+\left(\frac{6 \sin x \cos x\left(\cos ^{2} x-\sin ^{2} x\right)}{3 \sin ^{2} x \cos ^{2} x-2} v\right)^{\prime \prime}
$$

$$
-\left(\frac{9 \sin ^{2} x \cos ^{2} x+14}{3 \sin ^{2} x \cos ^{2} x-2} v\right)^{\prime}=0
$$

has a fundamental set of solutions $\sin ^{2} 2 x / W,\left(2 \sin x-3 \sin ^{3} x\right) / W$, and $\left(3 \cos ^{3} x-2 \cos x\right) / W$. It is easily confirmed that $\eta_{1}(0)=\eta_{2}(0)=\pi / 2$ for $(2.1)^{*}$ and no extremal solution for $\left[0, \eta_{1}(0)\right]$ of $(2.1)^{*}$ vanishes in $\left(0, \eta_{1}(0)\right)$. This shows that $\left(\mathrm{P}_{2}\right)$ does not in general imply $\left(\mathrm{P}_{1}\right)$.

To see that $\left(\mathrm{P}_{1}\right)$ does not in general imply $\left(\mathrm{P}_{2}\right)$, consider the nonsingular equation

$$
\left(6 x^{2}-8 x+3\right) y^{(v)}-(12 x-8) y^{\prime \prime \prime}+12 y^{\prime \prime}=0,
$$

for which $1, x, x(1-x)^{2}$, and $x(1-x)^{3}$ form a fundamental set of solutions, $\eta_{1}(0)=1$, and of which no extremal solution for $[0,1]$ has a 3-1 distribution of zeros [4]. Moreover, no extremal solution for [0, 1] can vanish more than once in $(0,1)$. It is easily verified that $(2.2)$ has no nontrivial solution with zeros of order 2 and 3 at $x=0$ and $x=1$, respectively. From these facts we can readily deduce $\eta_{1}(0)<$ $\eta_{2}(0)$. On the other hand, $x(\lambda-x)(1-x)^{2}, 0<\lambda<1$, is an extremal solution for $[0,1]$ which vanishes at $\lambda, 0<\lambda<1$.

An obvious consequence of these examples is that $\left(P_{3}\right)$ is not in general implied by either $\left(\mathrm{P}_{1}\right)$ or $\left(\mathrm{P}_{2}\right)$ alone.

In view of Theorem 2.2, it is clear that any differential equation which satisfies $\left(\mathrm{P}_{3}\right)$ will also satisfy $\left(\mathrm{P}_{1}\right)$ and $\left(\mathrm{P}_{2}\right)$. Consider a differential equation of the form

$$
L_{n} y+p y=0,
$$

where the operator $L_{n}$ is successively defined by 


$$
L_{0} y=\rho_{0} y, \quad L_{k} y=\rho_{k}\left(L_{k-1} y\right)^{\prime}, \quad k=2,3, \cdots, n .
$$

The functions $\rho_{0}, \rho_{1}, \cdots, \rho_{n}$ are assumed to be positive, $\rho_{k} \in C^{n-k}$, $k=0,1, \cdots, n$, and $p$ is assumed not to vanish. Eq. (2.3) was extensively studied by Nehari [7], who established the following result: If a nontrivial solution of (2.3) has zeros of order $k$ and $n-k$ at $x=a$ and $x=b$, respectively $(a<b)$, then $n-k$ is even or odd, according as $p<0$ or $p>0$. Evidently, this result implies that Eq. (2.3) satisfies $\left(\mathrm{P}_{3}\right)$. Hence, we have the following theorem.

THEOREM 2.3. Eq. (2.3) has the properties $\left(\mathrm{P}_{1}\right),\left(\mathrm{P}_{2}\right)$, and $\left(\mathrm{P}_{3}\right)$.

3. Zero distribution and factorization. In this section we exclusively consider a differential equation of the form (1.1) with property $\left(\mathrm{P}_{1}\right)$. Let $Y$ be an extremal solution of $(1.1)$ for $\left[a, \eta_{1}(a)\right]$ with an $i_{1}-i_{2}-\cdots-i_{j}$ distribution of zeros, $a=x_{1}<x_{2}<\cdots<x_{j}=\eta(a)$, $i_{1}+i_{2}+\cdots+i_{j}=n$. Then $Y$ has a zero of order exactly $i_{k}$ at $x_{k}, k=$ $1,2, \cdots, j$. This is because $\eta_{1}(a)<\eta_{2}(a)$. Therefore, by a repeated application of Theorem 2.1, we obtain

THEOREM 3.1. Suppose (1.1) has the property $\eta_{1}(a)<\eta_{2}(a)$ and has an extremal solution for $\left[a, \eta_{1}(a)\right]$ with an $i_{1}-i_{2}-\cdots-i_{j}$ distribution of zeros, $i_{1}+i_{2}+\cdots+i_{j}=n$. Let $k_{1}, k_{2}, \cdots, k_{p}$ be arbitrary positive integers such that $k_{1}+k_{2}+\cdots+k_{p}=n$, and let $a=\xi_{1}, \xi_{2}, \cdots, \xi_{p}=\eta_{1}(a)$ be distinct points in $\left[a, \eta_{1}(a)\right]$. If $i_{1} \leqq k_{1}$ and $i_{1} \leqq k_{p}$, then (1.1) has an extremal solution for $\left[a, \eta_{1}(a)\right]$ which has a zero of order exactly $k_{m}$ at $\xi_{m}, m=1,2, \cdots, p$.

As is clear from Theorem 3.1, the zeros of solutions in $\left(a, \eta_{1}(a)\right)$ can be moved to an arbitrary point in $\left(a, \eta_{1}(a)\right)$, or can be separated into lower-order zeros in $\left(a, \eta_{1}(a)\right)$. However, no such statements can be made in general for the zeros at the end points $a$ and $\eta_{1}(a)$. On the other hand, the zeros of an extremal solution for $\left[a, \eta_{1}(a)\right]$ can be simultaneously separated into simple zeros in $\left[a, \eta_{1}(a)+\epsilon\right), \epsilon>0$ $[4,14]$. By using a slight modification of the arguments given in the proof of Theorem 1 in [4], we shall establish the following result.

THEOREM 3.2. If (1.1) has a nontrivial solution with an $(n-l)-l$ distribution of zeros in $\left(a, \eta_{2}(a)\right)$, then (1.1) has a nontrivial solution with the zero distribution

$$
\underbrace{1-1-\cdots-1}_{i}-j-1 \underbrace{-\cdots-1,}_{k} \quad i+j+k=n,
$$

in $\left(a, \eta_{2}(a)\right)$, provided $i \geqq n-l$ or $k \geqq l$. 
Proof. Consider the case $i \geqq n-l$. Let $y$ be a nontrivial solution of (1.1) which has zeros of order $n-l$ and $l$ at $x=b$ and $x=c$, respectively, $a<b<c<\eta_{2}(a)$ and suppose $l$ is maximal. Consider the function

$$
w(x) \equiv\left\{\begin{array}{cc}
w\left(x ; c^{[n-1]}\right) & \text { if } \quad l=n-1 \\
w\left(x ; b^{[n-1-1]}, c^{[l]}\right), \text { otherwise }
\end{array}\right.
$$

defined in $\S 1$. This function $w$ cannot vanish identically; for if $w \equiv 0$ it would imply the existence of a nontrivial solution with a zero of order $n-l-1$ at $b$ and a zero of order $l+1$ at $c$, contrary to the assumption. Therefore, $w$ is a nontrivial solution of (1.1) with a zero of order exactly $n-l$ at $b$ and a zero of order exactly $l$ at $c$. Consequently, the $n-l$ zeros at $b$ and $l-j$ (out of $l$ ) zeros at $c$ can be separated into $n-j$ simple zeros in such a way that there are $i$ simple zeros to the left and $k$ simple zeros to the right of the $j$ th-order zero at $c$ (Cf. The proof of Theorem 1 [4]). This proves the theorem for the case $i \geqq n-l$.

The proof for the case $k \geqq l$ is similar.

REMARK. The above theorem can be restated as follows: If (1.1) does not have a nontrivial solution with the zero distribution (3.1) in $\left(a, \eta_{2}(a)\right)$, then (1.1) does not have nontrivial solutions in $\left(a, \eta_{2}(a)\right)$ with zero distributions $(n-1)-1,(n-2)-2, \cdots,(n-k)-k,(n-k-j)-$ $(k+j), \cdots, 1-(n-1)$.

We shall see that this result provides a link between the zero distribution and the factorization of the differential operator $L$ in (1.1). define

Let $y_{1}, y_{2}, \cdots, y_{n}$ be $n$ linearly independent solutions of (1.1) and

$$
W_{k} \equiv\left|\begin{array}{cccc}
y_{1} & y_{2} & \cdots \cdots & y_{k} \\
y_{1}^{\prime} & y_{2}^{\prime} & \cdots \cdots & y_{k}^{\prime} \\
& \ldots \ldots \cdots \cdots \cdots \cdots & \\
y_{1}^{(k-1)} & y_{2}^{(k-1)} & \cdots \cdots & y_{k}^{(k-1)}
\end{array}\right|, \quad k=1,2, \cdots, n .
$$

It is well-known that $W_{p}>0$ if and only if the operator $L$ can be written as $L=L_{1} L_{2}$, where $L_{1}$ and $L_{2}$ are nonsingular differential operators of order $n-p$ and $p$, respectively [15]. We require the following obvious extension of this result. 
THEOREM 3.3. Eq. (1.1) has $k$ solutions $y_{1}, y_{2}, \cdots, y_{k}$ such that $W_{k_{1}}>0, W_{k_{2}}>0, \cdots, W_{k_{1}}>0, k_{1}<k_{2}<\cdots<k_{l}=k$, if and only if the differential operator $L$ in (1.1) can be written as the product of $l+1$ nonsingular differential operators, i.e., $L=L_{l+1} L_{1} \cdots L_{1}$, where $L_{1}$ is of order $k_{1}, L_{i}$ is of order $k_{i}-k_{i-1}, i=2,3, \cdots, l$, and $L_{l+1}$ is of order $n-k_{l}$.

Suppose (1.1) does not have a nontrivial solution with an $(n-p)-$ $p$ distribution of zeros in $(a, b)$. Let $y_{1}, y_{2}, \cdots, y_{n}$ be solutions of (1.1) such that $y_{i}^{(n-j)}(a+\epsilon)=\delta_{i j}, \epsilon>0, i, j=1,2, \cdots, n$. Then $W_{p}>0$ in $(a+\epsilon, b)$. Since $\epsilon>0$ is arbitrary, we may assume that $W_{p}>0$ in $(a, b)$. Hence, we have $L=L_{1} L_{2}$, where $L_{1}$ and $L_{2}$ are nonsingular differential operators of order $n-p$ and $p$, respectively.

Likewise, from Theorems 3.2, 3.3, and the above remark we deduce

THEOREM 3.4. If (1.1) does not have a nontrivial solution with the zero distribution (3.1) in $\left(a, \eta_{2}(a)\right)$, the differential operator $L$ can be written as the product of nonsingular differential operators,

$$
L=L_{i+k+1} L_{i+k} \cdots L_{1}
$$

in $\left(a, \eta_{2}(a)\right)$, where $L_{m}, m \neq k+1$, is of first order and $L_{k+1}$ is of $j$ th order.

Let

$$
\mathfrak{Q} v=\sum_{k=0}^{n} q_{k}(\xi) v^{(k)}=0
$$

be the differential equation obtained from $L y=0$ through the change of variable $\xi=a+\eta_{2}(a)-x$. Clearly, $L y=0$ has a nontrivial solution with an $i_{1}-i_{2}-\cdots-i_{k}$ distribution of zeros in $\left(a, \eta_{2}(a)\right)$ if and only if $\mathfrak{L} v=0$ has a nontrivial solution with an $i_{k}-i_{k-1}-\cdots-i_{1}$ distribution of zeros in $\left(a, \eta_{2}(a)\right)$. In particular, if $L y=0$ does not have a nontrivial solution with the zero distribution (3.1), then $\mathfrak{l} v=0$ does not have a nontrivial solution with the zero distribution

$$
\underbrace{1-1-\cdots-1}_{k}-j-1-\underbrace{\cdots \cdots}_{i}-1, \quad i+j+k=n,
$$

in $\left(a, \eta_{2}(a)\right)$. Apply Theorem 3.4 to the nonsingular differential operator $\mathfrak{L}: \mathfrak{Q}$ can be written as the product of nonsingular differential operators

$$
\mathfrak{L}=\dot{\mathfrak{L}}_{1+k+1} \dot{\mathfrak{L}}_{i+k} \cdots \dot{\mathfrak{L}}_{1}
$$


in $\left(a, \eta_{2}(a)\right)$, where $\dot{\mathfrak{Z}}_{p}, p \neq i+1$, is of first order and $\dot{\mathfrak{Z}}_{i+1}$ is of $j$ th order. Transform the equation $\mathfrak{Q} v=\dot{\mathfrak{Q}}_{i+k+1} \dot{\mathfrak{L}}_{1+k} \cdots \dot{\mathfrak{Q}}_{1} v=0$ back to $L y=0$ by substituting $x=a+\eta_{2}(a)-\xi$. Under this transformation each differential operator $\dot{2}_{p}, p=1,2, \cdots, i+k+1$, in (3.2) remains nonsingular. Moreover, the order of each $\dot{\mathfrak{L}}_{p}$ and the order in which these differential operators appear remain unchanged. We summarize this result in the following theorem.

THEOREM 3.5. If (1.1) does not have a nontrivial solution with the zero distribution (3.1) in $\left(a, \eta_{2}(a)\right)$, the differential operator $L$ can be written as the product of nonsingular differential operators, $L=$ $\mathfrak{R}_{1+k+1} \mathfrak{R}_{1+k} \cdots \mathfrak{R}_{1}$, in $\left(a, \eta_{2}(a)\right)$, where $\mathfrak{Q}_{p}, p \neq i+1$, is of first order and $\mathfrak{L}_{1+1}$ is of $j$ th order.

\section{REFERENCES}

1. J. H. Barrett, Oscillation theory of ordinary linear differential equations, Advances in Mathematics, 3 (1969), 415-509.

2. G. B. Gustafson, Interpolation between consecutive conjugate points of an nth order linear differential equations, Trans. Amer. Math. Soc., 177 (1973), 237-255.

3. P. Hartman, Unrestricted n-parameter families, Rend. Circ. Mat. Palermo, 7 (1958), 123-142.

4. W. J. Kim, Simple zeros of solutions of nth-order linear differential equations, Proc. Amer. Math. Soc., 28 (1971), 557-561.

5. - On the extremal solutions of nth-order linear differential equations, Proc. Amer. Math. Soc., 33 (1972), 62-68.

6. W. Leighton and Z. Nehari, On the oscillation of solutions of self-adjoint linear differential equations of the fourth order, Trans. Amer. Math. Soc., 89 (1958), 325-377.

7. Z. Nehari, Disconjugate linear differential operators, Trans. Amer. Math. Soc., 129 (1967), $500-516$.

8. Z. Opial, On a theorem of O. Arama, J. Differential Equations, 3 (1967), 88-91.

9. A. C. Peterson, Distribution of zeros of solutions of a fourth order differential equation, Pacific J. Math., 30 (1969), 751-764.

10. The distribution of zeros of extremal solutions of a fourth order differential equation for the nth conjugate point, J. Differential Equations, 8 (1970), 502-511.

11. A. C. Peterson, On a relation between a theorem of Hartman and a theorem of Sherman, Canad. Math. Bull., 16 (1973), 275-281.

12. T. '. Sherman, Properties of solutions of nth order linear differential equations, Pacific J. Math., 15 (1965), 1045-1060.

13. - On a theorem of Azbelev and Caljuk, Proc. Amer. Math. Soc., 21 (1969), 63.

14. Conjugate points and simple zeros for ordinary linear differential equations, Trans.

Amer. Math. Soc., 146 (1969), 397-411.

15. A. Zettl, Factorization of differential operators, Proc. Amer. Math. Soc., 27 (1971), 425-426.

Received February 5, 1974 and in revised form June 14, 1974.

State University of New York - Stony Brook 


\section{PACIFIC JOURNAL OF MATHEMATICS \\ EDITORS}

\author{
Richard ARENS (Managing Editor) \\ University of California \\ Los Angeles, California 90024
}

\author{
R. A. Beaumont \\ University of Washington \\ Seattle, Washington 98105
}

\section{J. DugundJI}

Department of Mathematics

University of Southern California

Los Angeles, California 90007

D. Gilbarg and J. Milgram

Stanford University

Stanford, California 94305

\section{ASSOCIATE EDITORS}
E. F. BECKENBACH
B. H. NeumanN
F. WOLF
K. YoshidA

\section{SUPPORTING INSTITUTIONS}

\author{
UNIVERSITY OF BRITISH COLUMBIA \\ CALIFORNIA INSTITUTE OF TECHNOLOGY \\ UNIVERSITY OF CALIFORNIA \\ MONTANA STATE UNIVERSITY \\ UNIVERSITY OF NEVADA \\ NEW MEXICO STATE UNIVERSITY \\ OREGON STATE UNIVERSITY \\ UNIVERSITY OF OREGON \\ OSAKA UNIVERSITY
}

\author{
UNIVERSITY OF SOUTHERN CALIFORNIA \\ STANFORD UNIVERSITY \\ UNIVERSITY OF TOKYO \\ UNIVERSITY OF UTAH \\ WASHINGTON STATE UNIVERSITY \\ UNIVERSITY OF WASHINGTON \\ AMERICAN MATHEMATICAL SOCIETY
}

The Supporting Institutions listed above contribute to the cost of publication of this Journal, but they are not owners or publishers and have no responsibility for its contents or policies.

Mathematical papers intended for publication in the Pacific Journal of Mathematics should be in typed form or offset-reproduced (not dittoed), double spaced with large margins. Underline Greek letters in red, German in green, and script in blue. The first paragraph or two must be capable of being used separately as a synopsis of the entire paper. Items of the bibliography should not be cited there unless absolutely necessary, in which case they must be identified by author and Journal, rather than by item number. Manuscripts, in duplicate, may be sent to any one of the four editors. Please classify according to the scheme of Math. Reviews, Index to Vol. 39. All other communications should be addressed to the managing editor, or Elaine Barth, University of California, Los Angeles, California, 90024.

100 reprints are provided free for each article, only if page charges have been substantially paid. Additional copies may be obtained at cost in multiples of 50 .

The Pacific Journal of Mathematics is issued monthly as of January 1966. Regular subscription rate: $\$ 72.00$ a year (6 Vols., 12 issues). Special rate: $\$ 36.00$ a year to individual members of supporting institutions.

Subscriptions, orders for back numbers, and changes of address should be sent to Pacific Journal of Mathematics, 103 Highland Boulevard, Berkeley, California, 94708.

PUBLISHED BY PACIFIC JOURNAL OF MATHEMATICS, A NON-PROFIT CORPORATION Printed at Jerusalem Academic Press, POB 2390, Jerusalem, Israel.

$$
\begin{gathered}
\text { Copyright (c) } 1975 \text { Pacific Journal of Mathematics } \\
\text { All Rights Reserved }
\end{gathered}
$$




\section{Pacific Journal of Mathematics}

Vol. 56, No. 2 December, 1975

Ralph Alexander, Generalized sums of distances .................. 297

Zvi Arad and George Isaac Glauberman, A characteristic subgroup of a group of odd order ............................... 305

B. Aupetit, Continuité du spectre dans les algèbres de Banach avec involution ........................................ 321

Roger W. Barnard and John Lawson Lewis, Coefficient bounds for some classes of starlike functions . ...........................

Roger W. Barnard and John Lawson Lewis, Subordination theorems for some classes of starlike fumctions . .................... 333

Ladislav Bican, Preradicals and injectivity .................. 367

James Donnell Buckholtz and Ken Shaw, Series expansions of analytic

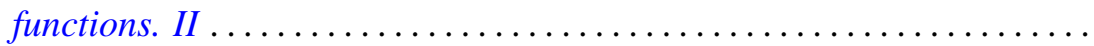

Richard D. Carmichael and E. O. Milton, Distributional boundary values in

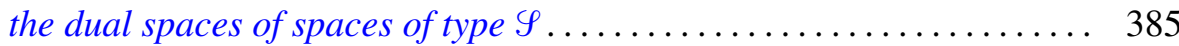

Edwin Duda, Weak-unicoherence ............................ 423

Albert Edrei, The Padé table of functions having a finite number of essential singularities ........................................

Joel N. Franklin and Solomon Wolf Golomb, A function-theoretic approach to the study of nonlinear recurring sequences ................ 455

George Isaac Glauberman, On Burnside's other $p^{a} q^{b}$ theorem 469

Arthur D. Grainger, Invariant subspaces of compact operators on topological vector spaces .........................

Jon Craig Helton, Mutual existence of sum and product integrals .

Franklin Takashi Iha, On boundary functionals and operators with finite-dimensional null spaces

Gerald J. Janusz, Generators for the Schur group of local and global number fields

A. Katsaras and Dar-Biau Liu, Integral representations of weakly compact operators.

W. J. Kim, On the first and the second conjugate points .

Charles Philip Lanski, Regularity and quotients in rings with involution ....

Ewing L. Lusk, An obstruction to extending isotopies of piecewise linear manifolds.

Saburou Saitoh, On some completenesses of the Bergman kernel and the Rudin kernel..

Stephen Jeffrey Willson, The converse to the Smith theorem for $Z_{p}$-homology spheres. 